\title{
Diseño y Construcción de una Máquina de Wimshurst para La Enseñanza de la Electrostática
}

\author{
Carlos A. Collazos ${ }^{(1)^{*}}$, Heindel R. Otero(2), Jaime J. Isaza ${ }^{(2)}$ y César Mora ${ }^{(1)}$ \\ (1) Centro de Investigación en Ciencia Aplicada y Tecnología Avanzada del Instituto Politécnico Nacional, \\ Av. Legaria 694, Col. Irrigación, C. P. 11500, México DF, México (e-mail: cacollazos@gmail.com, \\ ceml36@gmail.com) \\ (2) Departamento de Ciencias Naturales, Escuela Colombiana de Ingeniería, Ak. 45 No. 205-59, Autopista \\ Norte, Bogotá D. C., Colombia. (e-mail: heindel1297@gmail.com, jisazaceballos@gmail.com)
}

* Autor a quien debe ser dirigida la correspondencia

Recibido Feb. 2, 2016; Aceptado Abr. 4, 2016; Versión final Jun. 4, 2016, Publicado Oct. 2016

\section{Resumen}

En este trabajo se presenta el diseño y construcción de un prototipo de una Máquina de Wimshurst, explicando cuales son las partes importantes que conforman la máquina y el principio físico de su funcionamiento. El prototipo fue empleado para la enseñanza de la Electrostática en cursos de laboratorio de Electricidad y Magnetismo en la Escuela Colombiana de Ingeniería. El prototipo se usó con dos enfoques. El primer enfoque se direcciona a que los estudiantes realicen prácticas demostrativas a nivel de descargas eléctricas controladas y péndulo electrostático. El segundo enfoque se orienta a que los estudiantes experimenten y obtengan resultados cuantitativos para un laboratorio a nivel de Campo y Potencial Eléctrico. Los resultados muestran una ganancia de $0.52 \pm 0.08$ y corresponde a un rango de ganancia medio (entre 0.3 y 0.7 ). Se presenta la secuencia didáctica que siguen los estudiantes cuando realizan los laboratorios con el prototipo construido. En este trabajo también se describe el método usado y los instrumentos que se emplearon para el proceso de obtención de datos. Así mismo el trabajo presenta y discute las fortalezas de la estrategia empleada.

Palabras clave: máquina de Wimshurst; electrostática; enseñanza; práctica de laboratorio

\section{Design and Construction of a Wimshurst Machine for Teaching Electrostatic}

\begin{abstract}
In this work, the design and construction of a prototype for a Wimshurst machine is presented. The article indicates the important parts that make up the machine and the physical operating principle. The prototype was used for teaching Electrostatics in the laboratory of Electricity and Magnetism in the Colombian School of Engineering in the second half of 2015. The prototype was used with two purposes. The first purpose has the intention that students develop demonstrative practices according to level controlled electric shock and electrostatic pendulum practices. The second purpose is aimed at students to experience and obtain quantitative results for a laboratory level of Potential and Electric Field. The results show a gain $(0.52 \pm 0.08)$ and it corresponds to an average gain (between 0.3 y 0.7$)$. The didactic sequence followed by students when they work in the laboratory with the prototype. Also, in this paper the method used and the instruments used for data collection process are described. The paper presents and discusses the strengths of the strategy employed.
\end{abstract}

Keywords: Wimshurst machine; electrostatics; teaching; laboratory practice 


\section{INTRODUCCIÓN}

El uso de prototipos en la enseñanza de la física involucra a los estudiantes en un proceso experimental que les permite fortalecer conceptos y habilidades empíricas (Castellanos y D'Alessandro, 2003; Barbosa et al., 2011; Collazos et al., 2014; Valderrama y Valderrama, 2014). En particular para los cursos de laboratorio de Electricidad y Magnetismo, los fenómenos de electrostática son explicados por medio de prototipos como el Generador de Van der Graff, péndulo electrostático, electrodos de descarga, electróforos, Electroscopio, Máquina de Wimshurst y otros montajes experimentales (Ganci, 2009; Ganci y Ganci, 2012; Collazos y et al., 2016). Dentro de las máquinas electrostáticas se destaca la máquina Wimshurst por su complejidad en su diseño y construcción. Diferentes versiones de la máquina han sido construidas durante el transcurso de la Historia con base al diseño de James Wimshurst en 1883. A continuación se presentan las referencias más significativas sobre máquinas electrostáticas y algunos generadores de alta tensión. En (Simon,1926) se muestra en detalle la construcción de máquina de Wimshurst birotational donde se explica su principio físico de funcionamiento. En (Zahn et al,1974) se presenta una Máquina de Wimshurst de tres discos con su respectivo modelo físico además de un circuito electrónico equivalente. En (Aresti y Delunas,1983) se describe una máquina monodisco la cual según indican los autores puede ser menos eficiente que la máquina de Wimshurst pero más sencilla para explicar los principios de electrostática con los estudiantes. A pesar de que la invención de la máquina data del siglo XIX, existe una diversidad de máquinas de influencia que se basan en el principio original Wimshurst. En (De Queiroz, 2016) se lista 122 trabajos de máquinas que generan alta tensión y que tienen directa e indirectamente relación con la máquina de Wimshurst. El listado de Queiroz se acompaña de cortos comentarios de las principales publicaciones americanas como son: American Journal Physics; Physical Review, Review of Scientific Instruments entre otras publicaciones. A pesar de que los detalles técnicos de la máquina de Wimshurst son mostrados ampliamente en las referencias anteriores citadas, es importante resaltar que hay muy poco material pedagógico entorno al proceso de aprendizaje en que está inmerso un estudiante cuando construye o experimenta con este tipo de máquina.

Este trabajo presenta el diseño y construcción de una máquina de Wimshurst que genera una tensión eléctrica aproximadamente de un orden de magnitud de $10^{3} \mathrm{~V}$. Aunque la máquina es bastante popular, son pocas las fuentes bibliográficas que presentan simultáneamente las dimensiones, los materiales y su uso en la enseñanza de la electrostática. Este artículo indica aspectos claves en el diseño que pueden permitir reproducir la máquina más fácilmente tanto por estudiantes como docentes. El trabajo presenta cada una de las partes que conforman la máquina con sus dimensiones respectivas. El prototipo construido permite observar el efecto corona, las líneas de campo eléctrico y otros experimentos de electrostática. En el ámbito de la enseñanza de la electrostática la experimentación con la máquina involucra conceptos físicos relacionados con la inducción, fricción, conducción, fuerza y campo eléctrico, capacitancia y potencial eléctrico. El trabajo se ha estructurado de la siguiente manera: en la sección Diseño del prototipo se detalle técnicamente cada una de las partes de la máquina con sus respectivas dimensiones y materiales. La sección Experimentación con el prototipo y Valoración de la estrategia, indica los resultados de la aplicación de la secuencia didáctica que siguen los estudiantes en un laboratorio a nivel de descargas eléctricas controladas, péndulo electrostático y potencial y campo eléctrico.

\section{DISEÑO DEL PROTOTIPO}

Las Figuras 1 y 2 presentan dos fotografías con vistas superior y frontal de la máquina de Wimshurst construida. A continuación se describirán las partes principales de la máquina. Según indica la Figura 1 la máquina posee dos discos ( $\mathrm{A}$ y $\mathrm{A}^{\prime}$ ) de material aislante, en este caso acrílico. Los discos rotan en sentido contrario debido a un eje común longitudinal (B), el cual está sostenido por los tornillos (C y $\left.C^{\prime}\right)$ y una manivela (D). La separación entre los discos es de $1.510^{-3} \mathrm{~m}$. En la superficie de ambos discos unas láminas de aluminio (E) se han ubicado concéntricamente. Las Figuras 1 y 2 muestran las láminas para uno de los discos. La carga eléctrica se produce por fricción, en el momento en que las láminas entran en contacto con los peines de cobre $\left(F\right.$ y $\left.F^{\prime}\right)$. Los peines se han dispuesto en los extremos de las barras aisladas de aluminio $\left(G\right.$ y $\left.G^{\prime}\right)$. Las barras aisladas a su vez se mantienen de un soporte horizontal $(H)$. Los dos discos tienen un colector de carga $\left(\mathrm{H}_{\mathrm{y}} \mathrm{H}^{\prime}\right)$. A cada colector le corresponde un tipo de carga diferente.

La Figura 2 muestra que los colectores están conectados a su vez a dos botellas de Leyden (I y I'). Las botellas actúan como dos condensadores con una tierra común (J). La máquina es accionada por un sistema de poleas ( $\mathrm{K}$ y $\mathrm{K}^{\prime}$ ) con su respectiva banda y manivela (D). Las poleas están acopladas a los soportes de los discos (L). Los colectores de carga y las botellas de Leyden por su parte están conectados a un par de barras conductoras ( $\mathrm{M} \mathrm{y}^{\prime}$ ) donde en la parte terminal hay dos esferas ( $\mathrm{N}$ y $\mathrm{N}^{\prime}$ ), en las cuales es posible apreciar una descarga eléctrica cuando la máquina es accionada. Las barras conductoras se maniobran a su vez por dos manijas ( 0 y $0^{\prime}$ ) que permiten acercar o alejar las esferas. Todas las partes que conforman la máquina están ubicadas sobre una base aislada construida de madera $(\mathrm{P})$. 


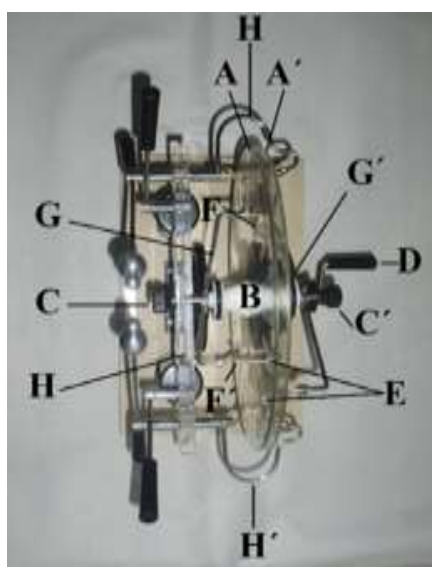

Fig. 1: Vista superior de la Máquina

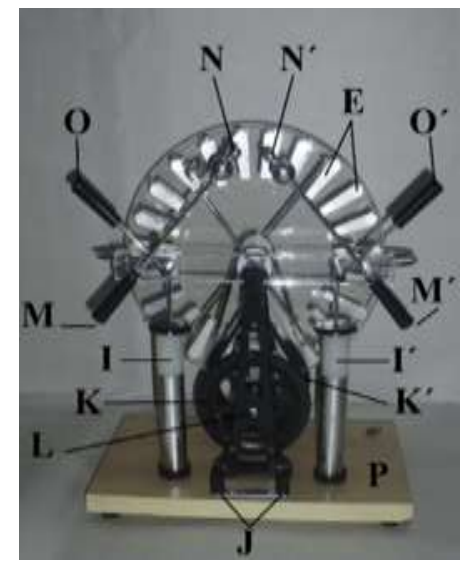

Fig. 2: Vista frontal de la Máquina

Discos $\left(A\right.$ y $\left.A^{\prime}\right)$ y Láminas $(E)$

Se conforma de dos discos de acrílico de diámetro de $25 \mathrm{~cm}$ y $0.3 \mathrm{~cm}$ de espesor. Los discos en el centro tienen un agujero circular de $1 \mathrm{~cm}$ de diámetro. Cada uno de los discos tiene 23 láminas de aluminio de $4 \mathrm{~cm}$ de largo y $0.7 \mathrm{~cm}$ de ancho con bordes terminados en punta como se indica en la Figura 3.

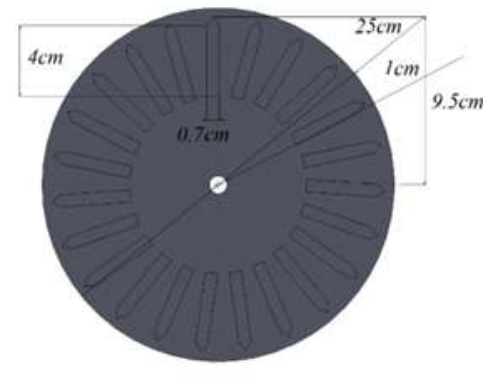

Fig. 3: Disco y láminas

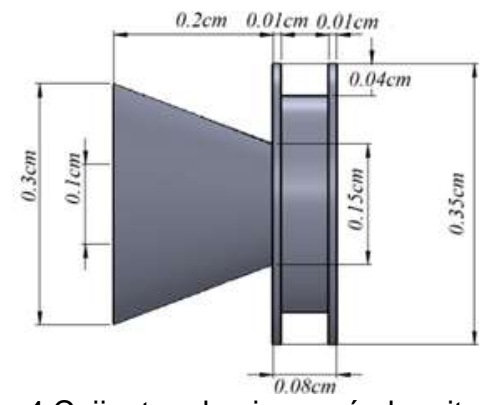

Fig.4:Cojinetes de eje común longitudinal

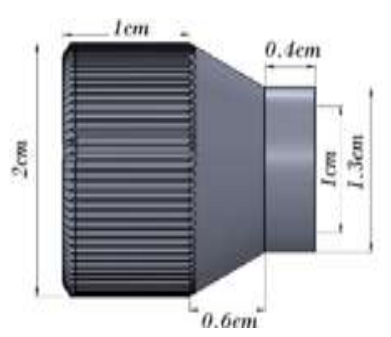

Fig. 5: Tornillos

Eje Común Longitudinal (B) y Tornillos (C y $\left.C^{\prime}\right)$

El eje común longitudinal consta de dos cojinetes de plástico que acoplan los dos discos ( $A$ y $\left.A^{\prime}\right)$. El cojinete tiene $3.0 \mathrm{~cm}$ de diámetro y $2.8 \mathrm{~cm}$ de largo. En el centro tiene un agujero de $1.5 \mathrm{~cm}$ de diámetro como se indica en la Figura 4. Los cojinetes son fijados por un par de tornillos plásticos de diámetro interno de $1 \mathrm{~cm}$ y diámetro externo de $1.3 \mathrm{~cm}$ según indica la Figura 5.

\section{Soporte Horizontal $(H)$}

El soporte es de acrílico y tiene $25 \mathrm{~cm}$ de largo y $2.5 \mathrm{~cm}$ de alto. Posee 5 orificios de $1 \mathrm{~cm}$ de diámetro, ubicados como se indica en la Figura 6. El orificio central permite incrustar el eje común longitudinal y hace que los discos giren en sentido contrario.

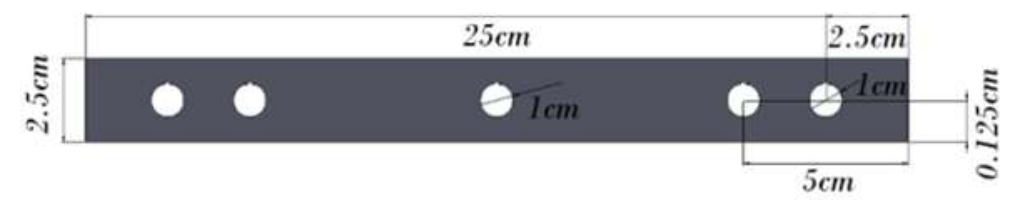

Fig.6: Soporte Horizontal

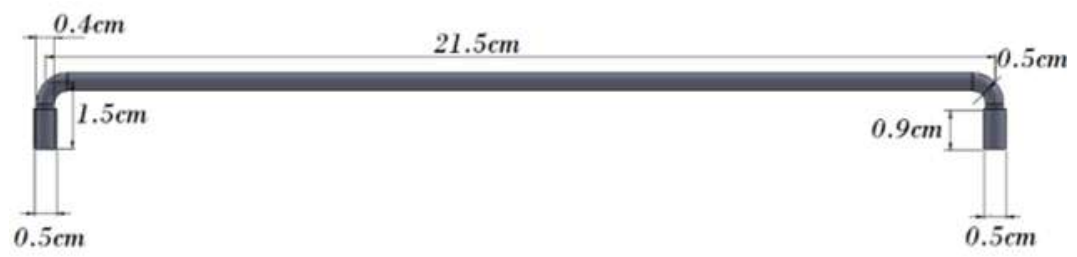

Fig. 7: Barra aislada 


\section{Barra Aislada (G y $G^{\prime}$ )}

Son dos varillas de aluminio de $21.5 \mathrm{~cm}$ como se indica en la Figura 7 . Las varillas tienen en los extremos los peines metálicos de cobre.

Botella de Leyden (I y I')

Son dos botellas en acrílico de diámetro de $2.5 \mathrm{~cm}$ y alto de $13 \mathrm{~cm}$. Posee una tapa plástica de $4 \mathrm{~cm}$ de diámetro y una varilla de aluminio de $17 \mathrm{~cm}$, la cual conecta al colector de carga según indica la Figura 8 . La parte exterior de los cilindros que conforman las botellas están recubiertas de papel aluminio.

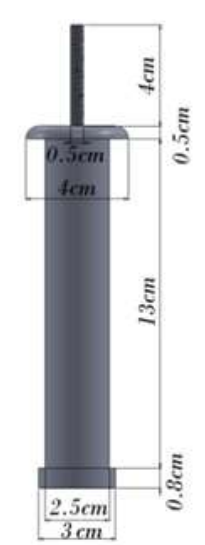

Fig. 8: Botella de Leyden

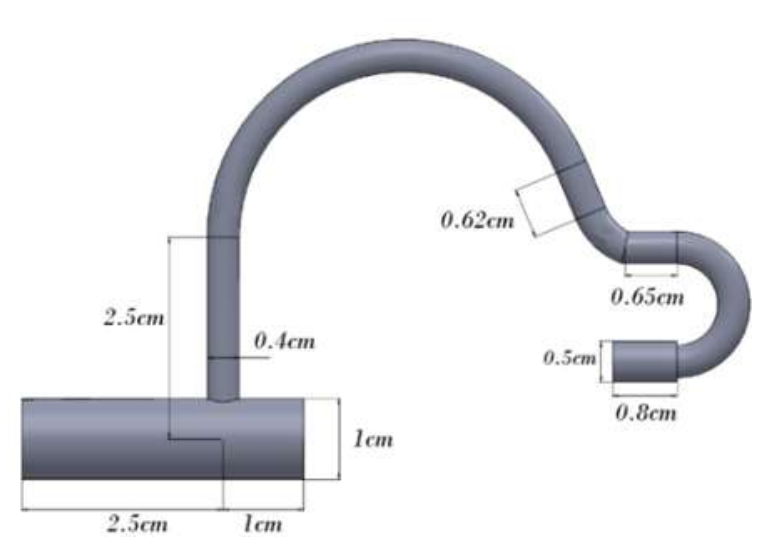

Fig. 9: Colectores de Carga

Colectores de carga $\left(H\right.$ y $\left.H^{\prime}\right)$

Son un par de alambres de aluminio doblados en $U$, donde en su parte terminal se ubican los peines de cobre. Posee un mango de aluminio que soporta toda la estructura curva. Los colectores están unidos al soporte horizontal por medio de los orificios de $1 \mathrm{~cm}$ de diámetro ubicados en los extremos como indica la Figura 9.

Varillas de Descarga (M y M') y Manijas (O y O')

Las varillas de descarga son de aluminio y tienen un largo de $11.5 \mathrm{~cm}$ con un mango aislante de $7 \mathrm{~cm}$. En la parte terminal de las varillas se dispone de 2 esferas. La esfera grande y pequeña tiene respectivamente $2 \mathrm{~cm}$ y $1 \mathrm{~cm}$ de diámetro como indica la Figura 10. Las dos manijas se acondicionan a las varillas de descarga. Las varillas tienen $7 \mathrm{~cm}$ de largo y un mango aislante de $2.5 \mathrm{~cm}$ de longitud como se representa en la Figura 11. La función de las manijas es acercar o alejar las varillas de descarga para percibir el efecto corona.

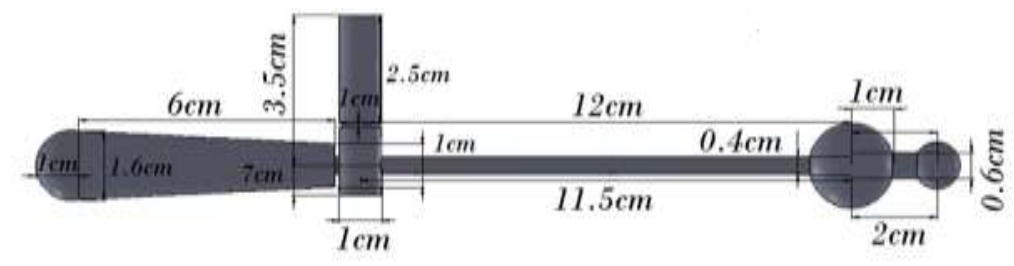

Fig.10: Varillas de Descarga

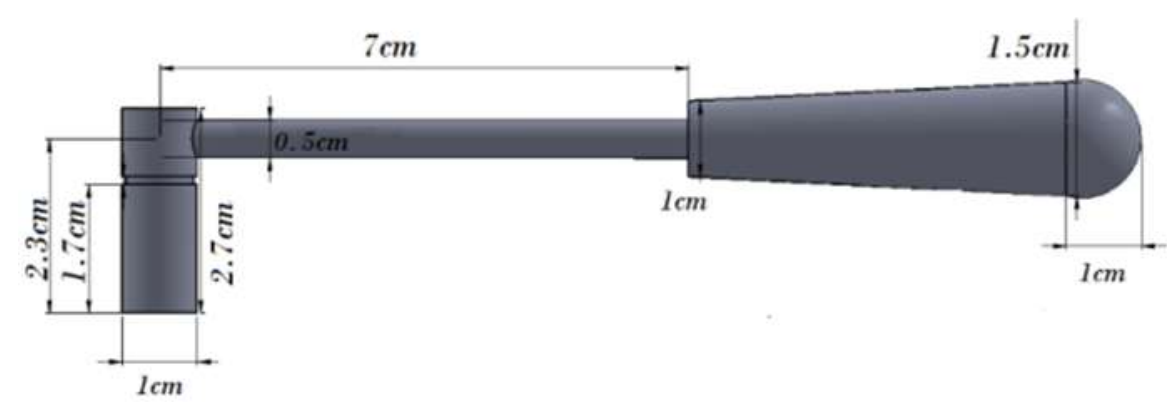

Fig. 11: Manijas 


\section{Soporte de Discos (L)}

Se dispone de dos soportes de discos de $15.9 \mathrm{~cm}$ de altura y de base $6.62 \mathrm{~cm}$, así como lo indica la Figura 12.

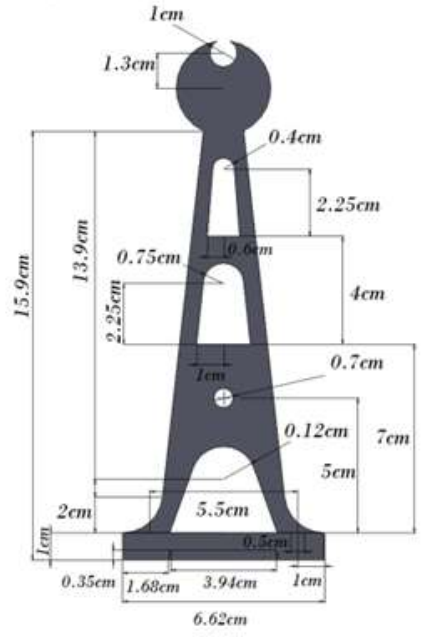

Fig.12: Soporte de Discos

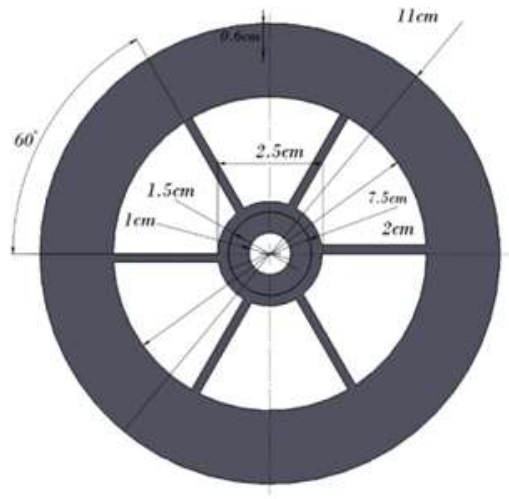

Fig. 13: Poleas

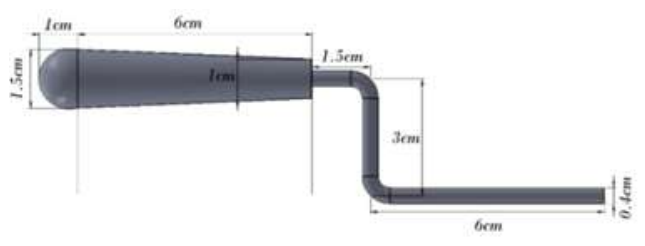

Fig. 14: Manivela

Poleas (KYK')

Sobre cada uno de los soportes de discos se ubican dos poleas cuyo diámetro interno es de $1 \mathrm{~cm}$ y diámetro externo es de $2 \mathrm{~cm}$, como se representa en la Figura 13.

Manivela (D)

Es una pieza de aluminio de largo $6 \mathrm{~cm}$ con un mango de plástico de $7 \mathrm{~cm}$ de longitud como se indica en la Figura 14. Esta pieza permite mover el disco $\left(\mathrm{A}^{\prime}\right)$ en sentido horario o antihorario.

\section{$\operatorname{Base}(P)$}

Soporte de madera compuesta de una base de $20 \mathrm{~cm}$ ancho y $30 \mathrm{~cm}$ de largo. En la Figura 15 se muestra la ubicación simétrica del soporte de los discos, las botellas de Leyden y la tierra común. La Figura 16 presenta el diseño en 3 dimensiones integrando cada una de los elementos anteriormente detallados.

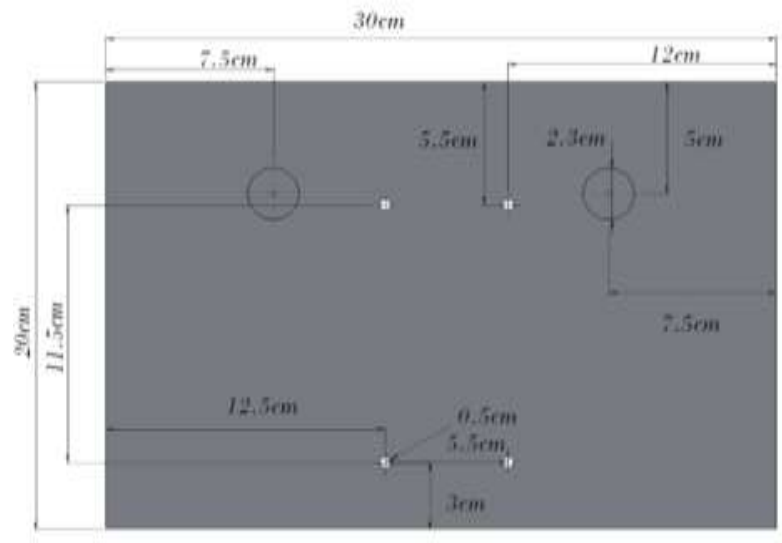

Fig. 15: Base

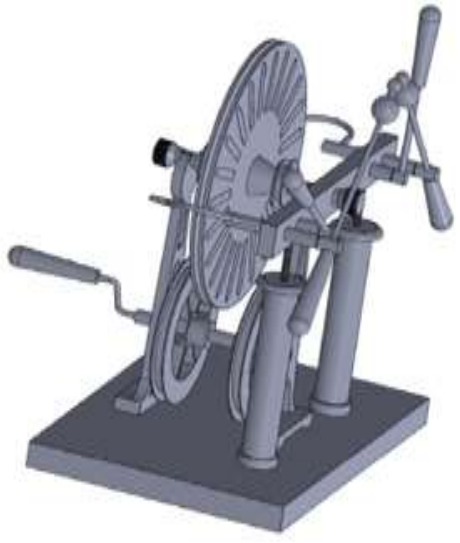

Fig. 16: Diseño en 3D de la Máquina

\section{EXPERIMENTACIÓN CON EL PROTOTIPO CONSTRUIDO Y VALORACIÓN DE LA ESTRATEGIA}

Para el proceso experimental con la máquina construida se empleó una guía de laboratorio, la cual puede ser descargada de fisicacollazos,2016. La guía fue aplicada en cursos de laboratorio de Electricidad y Magnetismo de la Escuela Colombiana de Ingeniería en el segundo semestre de 2015. Los estudiantes siguen una la secuencia didáctica para dos sesiones de laboratorio. La intensidad horaria de cada sesión es de 1.5 horas. En la primera sesión se aborda una práctica demostrativa a nivel de descargas eléctricas controladas y péndulo electrostático, además del concepto "líneas de campo eléctrico para diversos electrodos (cargas puntuales, laminas paralelas y anillos concéntricos)". 
En la segunda sesión se revisa el concepto de potencial eléctrico para los mismos electrodos usados en la sesión 1. La guía se compone de un preinforme e informe. El preinforme hace alusión a la actividad preliminar que deben responder los estudiantes grupalmente (como mínimo 3 estudiantes y como máximo 4 estudiantes). Para el preinforme los estudiantes deben plantear los objetivos de la práctica y responder grupalmente las 15 preguntas planteadas, las cuales son alusivas a los conceptos de descarga eléctrica, péndulo electrostático, fuerza, campo y potencial eléctrico, ley de Gauss y capacitancia. El cuestionario completo puede ser descargado de fisicacollazos, 2016.

Para el caso del informe se orienta el procedimiento experimental para que los estudiantes registren a mano los comentarios de los fenómenos demostrativos observados (Descargas eléctricas controladas y Péndulo Electrostático) y grafiquen las líneas de campo y potencial eléctrico. Los estudiantes reportan Tablas y gráficos y realizan cálculos, análisis y conclusiones. El informe tiene un valor del $80 \%$ con respecto al $20 \%$ del preinforme. Es importante resaltar que en el informe los estudiantes vuelven a resolver grupalmente las 15 preguntas planteadas inicialmente en el preinforme. Las preguntas se evalúan según el criterio de evaluación indicado en la guía y disponible en fisicacollazos, 2016. En este trabajo el cuestionario del preinforme fue considerado la prueba de entrada y el cuestionario del informe la prueba de salida una vez los estudiantes realizan el proceso experimental con la Máquina de Wimshurst. A continuación se describen cada uno de los experimentos realizados por los estudiantes y la valoración de estrategia aplicada en los estudiantes. Todo el material alusivo a la estrategia aplicada (cuestionario, guía de laboratorio, criterios de evaluación ) puede ser solicitado vía correo electrónico al autor que se señala para la correspondencia.

Laboratorios Demostrativos (Descargas eléctricas controladas, Péndulo Electrostático) y Líneas de Campo Eléctrico y Equipotenciales.

El prototipo presentado en este trabajo fue usado para realizar 4 experiencias demostrativas con los estudiantes. El primer experimento busca obtener una descarga eléctrica entre los terminales de la máquina como se indica en la Figura 17. El segundo experimento pide analizar los destellos de una lámpara fluorescente como se ilustra en la Figura 18. El tercer experimento usa una esfera metálica (cascabel) para que haga las veces de un péndulo y se ilustra en la Figura 19. El cuarto experimento plantea observar el comportamiento característico a nivel de líneas de campo eléctrico en un dipolo como se indica en la Figura 20.

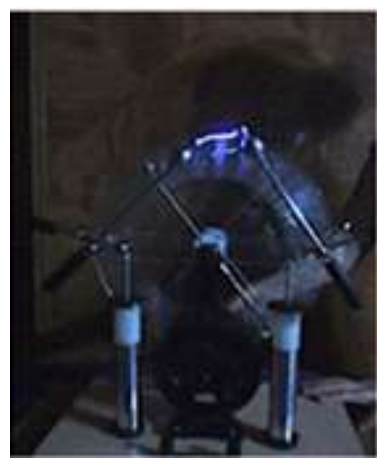

Fig. 17: Descarga

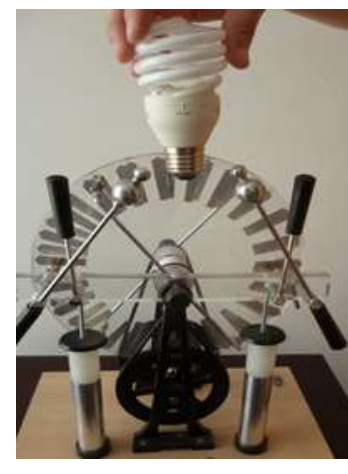

Fig. 18: Fluorescencia

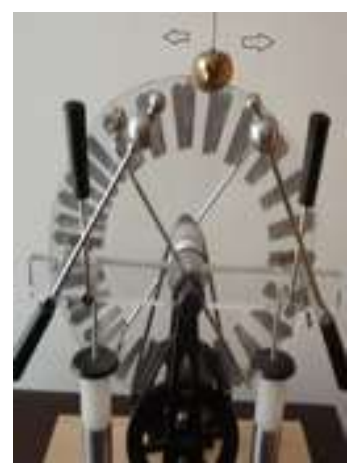

Fig. 19: Péndulo

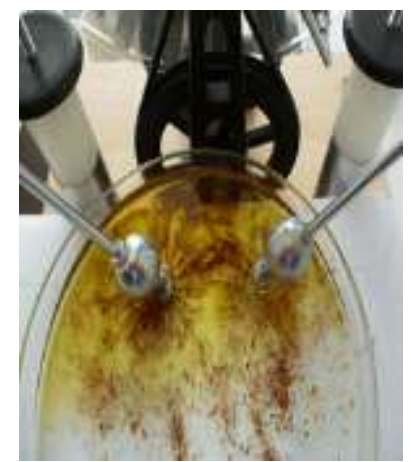

Fig. 20: Líneas de Campo

Para el quinto experimento el tema de líneas de campo eléctrico es extendido para placas paralelas y anillos concéntricos con un montaje experimental como el indicado en la figura 21 . El montaje involucra una cubeta con aceite donde están inmersos los electrodos, los cuales a su vez están energizados por la máquina de Wimshurst. Los estudiantes arrojan pequeñas cantidades de harina sobre el aceite para visualizar las líneas de campo en la cubeta o con el proyector de acetatos como se muestra en las figura 22 y 23 respectivamente.

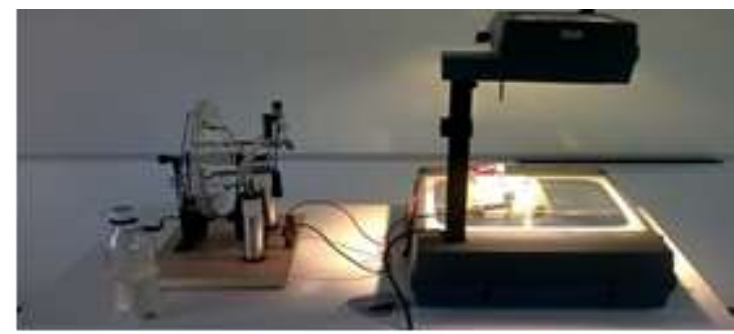

Fig. 21: Montaje experimental

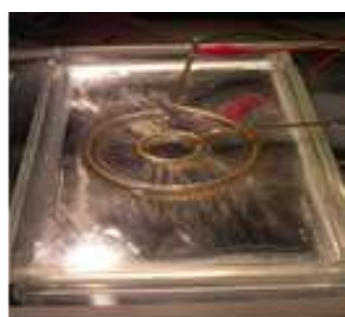

Fig. 22: Líneas de Campo

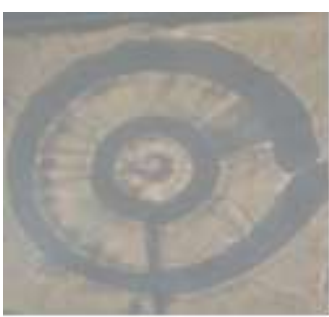

Fig. 23: Líneas proyectadas 
Para el sexto experimento en el montaje de la Figura 21 es necesario reemplazar la Máquina de Wimshurst por una fuente de voltaje DC y el aceite por agua. Por medio de un voltímetro los estudiantes registran los valores de potencial Eléctrico para los electrodos usados en el experimento 4 y 5. La Figura 24 indica los patrones de líneas equipotenciales respectivamente de un dipolo, placas paralelas y anillos concéntricos, además de las líneas campo eléctrico inferidas por los estudiantes. En físicacollazos, 2016 se presenta en detalle el procedimiento de cada uno de los experimentos.
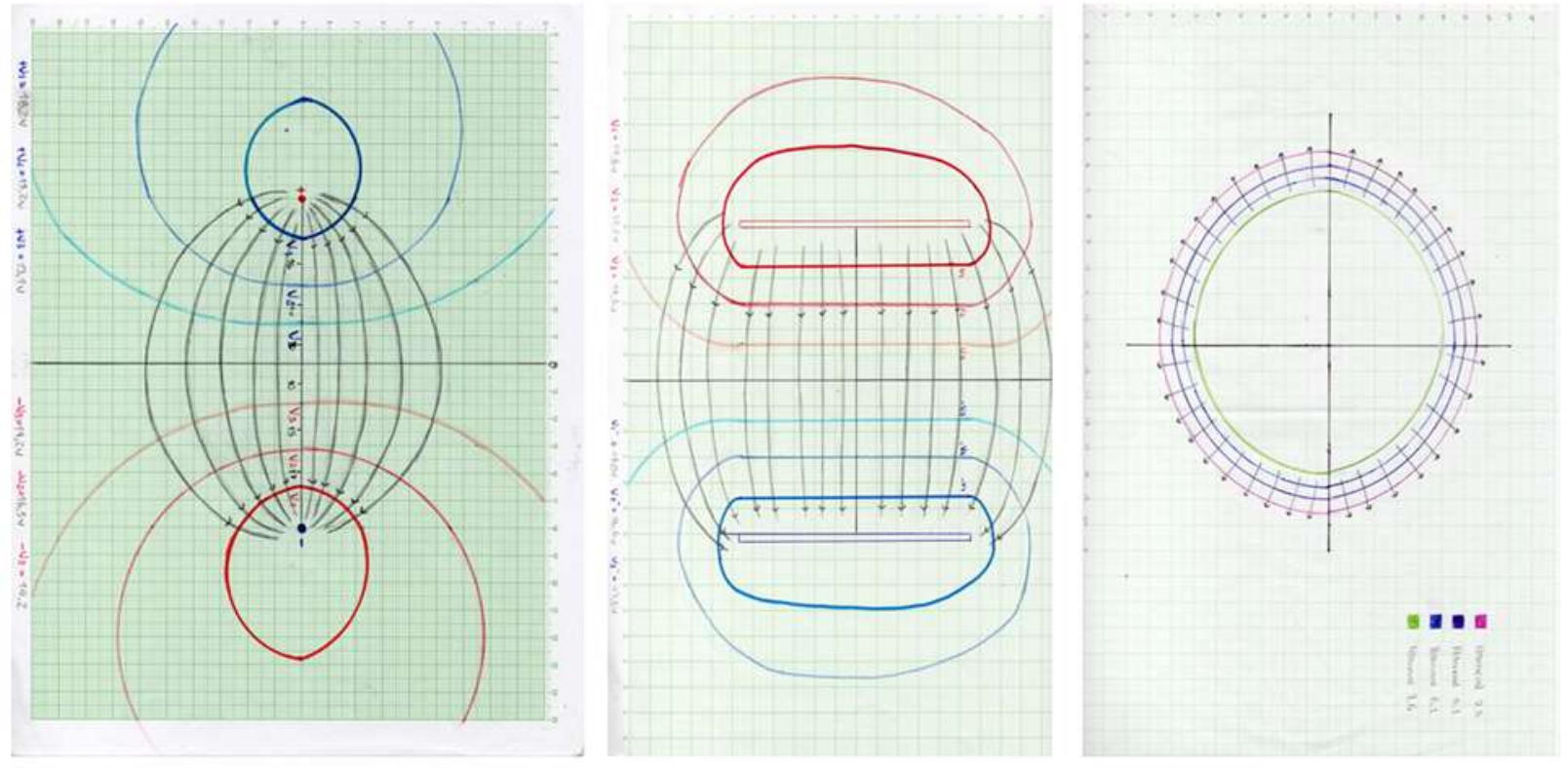

Fig. 24: Líneas campo Eléctrico y equipotenciales graficadas por estudiantes

\section{Análisis de Resultados del uso de la máquina de Wimshurst por los estudiantes}

El cuestionario se evalúa sobre 100 puntos y busca valorar conceptos físicos y definiciones para algunos tópicos como se indica a continuación: Pregunta 1: Potencial eléctrico en una Descarga; Pregunta 2: Efecto Corona; Pregunta 3: Ionización; Pregunta 4: Fuerza y Campo Eléctrico en un péndulo electrostático; Pregunta 5: Capacitancia en una botella de Leyden; Pregunta 6: Campo escalar; Pregunta 7: Campo vectorial; Pegunta 8: Campo eléctrico como campo vectorial; Pregunta 9: Potencial eléctrico como campo escalar; Pregunta 10: La relación geométrica entre las líneas de campo eléctrico y las equipotenciales; Pregunta 11: Efecto borde; Pregunta 12: El efecto borde en algunos electrodos (dipolo, placas paralelas y anillos concéntricos); Pregunta 13: La fuerza, campo y trabajo eléctrico y su relación con el potencial eléctrico; Pregunta 14: Capacitancia y dieléctrico; Pregunta 15: Ley de Gauss.

Tabla 1. Resultados promedio para $(\mathrm{Pi})$ y $(\mathrm{Pf})$ con sus respectivas ganancias para el cuestionario de 15 preguntas

\begin{tabular}{|c|c|c|c|}
\hline Pregunta & $\mu(\mathrm{Pi}) \pm \sigma(\mathrm{Pi})$ & $\mu(\mathrm{Pf}) \pm \sigma(\mathrm{Pf})$ & $\overline{\mathrm{g}}_{\mathrm{fi}}+\sigma_{\mathrm{g}_{\mathrm{fi}}}$ \\
\hline 1 & $45.23 \pm 4.23$ & $79.21 \pm 5.23$ & $0.62 \pm 0.06$ \\
\hline 2 & $43.32 \pm 3.21$ & $67.21 \pm 4.22$ & $0.42 \pm 0.05$ \\
\hline 3 & $40.21 \pm 5.23$ & $72.15 \pm 5.13$ & $0.53 \pm 0.04$ \\
\hline 4 & $50.42 \pm 3.14$ & $78.12 \pm 4.54$ & $0.55 \pm 0.06$ \\
\hline 5 & $50.22 \pm 4.18$ & $77.15 \pm 3.18$ & $0.54 \pm 0.04$ \\
\hline 6 & $55.60 \pm 4.17$ & $76.14 \pm 4.17$ & $0.46 \pm 0.06$ \\
\hline 7 & $56.01 \pm 4.38$ & $78.02 \pm 6.32$ & $0.50 \pm 0.04$ \\
\hline 8 & $49.21 \pm 5.23$ & $80.21 \pm 4.37$ & $0.61 \pm 0.06$ \\
\hline 9 & $48.92 \pm 4.32$ & $79.15 \pm 5.41$ & $0.59 \pm 0.05$ \\
\hline 10 & $50.27 \pm 5.18$ & $81.23 \pm 5.62$ & $0.62 \pm 0.03$ \\
\hline 11 & $38.15 \pm 3.73$ & $67.45 \pm 4.11$ & $0.47 \pm 0.04$ \\
\hline 12 & $40.12 \pm 4.80$ & $68.56 \pm 5.18$ & $0.47 \pm 0.05$ \\
\hline 13 & $42.16 \pm 4.92$ & $65.76 \pm 5.38$ & $0.41 \pm 0.03$ \\
\hline 14 & $49.22 \pm 5.23$ & $82.12 \pm 4.43$ & $0.65 \pm 0.04$ \\
\hline 15 & $38.25 \pm 4.57$ & $65.16 \pm 3.18$ & $0.43 \pm 0.05$ \\
\hline Consolidado & $46.48 \pm 5.88$ & $74.51 \pm 6.11$ & $0.52 \pm 0.08$ \\
\hline
\end{tabular}


La Tabla 1 presenta los resultados promedio $\mu$, la desviación estándar $\sigma$ y ganancia para el cuestionario de las 15 preguntas planteadas y para 4 grupos de laboratorio(83 estudiantes). La Tabla 1 indica que los estudiantes comienzan con un desempeño regular en el promedio de la calificación del cuestionario del preinforme $(\mathrm{Pi})$ y que corresponde a un valor de (46.48 \pm 5.88$)$. Puede notarse en la Tabla 1 , que en la evaluación del promedio para el cuestionario del informe (Pf) hay una mejora progresiva que corresponde a un valor de (74.51 \pm 6.11$)$. Con base en los resultados obtenidos se observa un cambio significativo entre el promedio de las evaluaciones $(\mathrm{Pi})$ y $(\mathrm{Pf})$. Este cambio fue cuantificado por medio de la ecuación de ganancia de Hake $\bar{g}_{\mathrm{fi}}+\sigma_{\mathrm{g}_{\mathrm{fi}}}$ (Hake, 1999). Una explicación y uso detallado de la ecuación de Hake es mostrada en (Collazos,2016). El valor de ganancia obtenido entre los resultados promedio de $(\mathrm{Pi})$ y $(\mathrm{Pf})$ fue de $(0.52 \pm 0.08)$ y corresponde a un rango de ganancia medio (entre 0.3 y 0.7$)$ según (Hake, 1999) . Las ganancias obtenidas en cada una las 15 preguntas indicadas en la Tabla 1 se ubican también en un rango de ganancia medio y muestran que los estudiantes tienen mejores argumentos para socializar y sustentar sus resultados una vez que realizan los experimentos propuestos, además muestran mejoras progresivas entre la entrega del preinforme e informe. Es crucial resaltar que el uso de la máquina de Wimshurst permite reforzar a nivel teórico y experimental principios como la cuantificación y la conservación de la carga, mecanismos para producción de cargas (fricción, inducción), observar fenómenos de ionización y descarga eléctricas controladas, trabajar con diversos tipos de materiales (conductores, aislantes, dieléctricos), reconocer la conducción y polarización de materiales, identificar características vectoriales del campo eléctrico y propiedades de tipo escalar para el potencial eléctrico. Adicionalmente permite aplicar la ley de Gauss a un contexto real y comprender el concepto de capacitancia y dieléctrico

\section{CONCLUSIONES}

La construcción y explicación técnica del funcionamiento de la Maquina de Wimshurst y los experimentos planteados durante su operación permiten usar la máquina al nivel de la enseñanza de la electrostática. Se pudo establecer que los estudiantes:

- Experimentaron con la máquina involucrando conceptos como carga eléctrica, repulsión entre cargas del mismo género y atracción de las opuestas, polarización en dieléctricos, conducción de carga y almacenamiento de carga.

-conceptualizaron tópicos como la inducción, la fricción, así mismo conceptos de fuerza, campo y potencial eléctrico, además de la ley de Gauss y el concepto de capacitancia.

- Involucraron empíricamente los principios fundamentales de la electrostática como son la conservación y la cuantificación de la carga, además del concepto de tierra y portadores de carga.

- Trabajaron colaborativamente en la respuesta del cuestionario planteado. La metodología empleada llevó a los estudiantes a responder las inquietudes propuestas por medio de validaciones experimentales. Al finalizar su informe de laboratorio realizaron un buen análisis e interpretación de los datos obtenidos.

- Fortalecieron su nivel de abstracción, en tanto que pudieron llevar conceptos a un contexto experimental. Se motivaron y activaron sus habilidades para observar, analizar y deducir.

\section{REFERENCIAS}

Aresti A., Delunas A, Two-inductor electrostatic generator, doi:10.1119/1.13478 Am. J. Phys. (en línea), 51(5), 472(1983)

Barbosa L. H., Mora C., Talero P., Organista J. O., El Soplador mágico: un experimento discrepante en el aprendizaje de la ley de presión hidrodinámica de Bernoulli, doi: 10.1590/S1806-11172011000400009, Revista Brasileira de Ensino de Física (en línea), 33(4), 1-7 (2011)

Castellanos, M.L. y A. D'Alessandro, Proyectos de Investigación: Una Metodología para el Aprendizaje Significativo de la Física en Educación Media, Rev. Pedagogía, ISSN 0798-9792 (en línea): 24(69), 101-136 2003,http://www.scielo.org.ve/scielo.php?script=sci_abstract\&pid=S079897922003000100005\&lng=es\&nrm =iso\&tlng=es. Acceso 15 de enero (2016)

Collazos, C. A., R. Otero, Isaza J. y Mora C., Teaching Of Physics Throught Of The Construction Of Prototypes, The GIREP-MPTL: International conference on Teaching/Learning Physics: Integrating Research into Practice, Palermo, Italia (2014) 
Collazos, C. A., R. Otero, Isaza J. y Mora C., Enseñanza de la Electrostática por Medio de la Construcción de Prototipos y el Aprendizaje Basado en Proyectos, doi:10.4067/S0718-50062016000200013,Formación Universitaria (en línea). 9(2), 115-122 (2016)

Collazos, C. A., fisicacollazos, Página Web de Carlos Collazos, http://www.fisicacollazos.260mb.com/?cat=46. Acceso: Junio (2016)

De Queiroz A. C. M., Electrostatic Machines- Papers, http://www.coe.ufrj.br/ acmq/epapers.html, Acceso 4 de Junio de (2016)

Ganci A., Ganci S., Demonstration experiments in electrostatics: low cost devices, doi: 10.1590/S1806-

11172012000200012, Revista Brasileira de Ensino de Física (en línea), 34(1), 1-14(2012)

Ganci S., Makers of scientic instruments in a little Italian town,doi: 10.1590/S1806-11172009005000003

Revista Brasileira de Ensino de Física: 31(3), 1-3(2009)

Hake, R., Interactive-engagement vs traditional methods: A six-thousand-student survey of mechanics test data for introductory physics courses, doi: 10.1119/1.18809, American Journal Physics (en línea), 66, 64-74 (1996)

Simon, A. W., On the Quantitative Theory of Electrostatic Systems, doi: 10.1103/PhysRev.26.111, Phys. Rev. (en línea), 28(1), 111-117 (1926)

Simon, A. W., On the Quantitative Theory of the Wimshurst Static Machine, doi: 10.1063/1.1749075, Rev. Sci. Instrum. (en línea), 4 (2), 67-74 (1933)

Valderrama A. J., Valderrama J. O., Trabajo Semestral Guiado: un Buen Complemento al Estudio y Aprendizaje, doi: 10.4067/SO71850062014000300002, Formación Universitaria (en línea), 7(3), 3-10 (2014)

Zahn, M., Goslin, R. L. and Wicks, L. F., Self- Excited, Alternating, High-Voltage Generation Using a Modified Electrostatic Influence Machine, doi: 10.1119/1.1987674, Am. J. Phys. (en línea) , 42 (4), 289-294 (1974) 
